\title{
Hemofilia adquirida. Presentación de caso clínico y revisión de la bibliografía.
}

\author{
Acquired hemophilia. Presentation of clinical case and review of the \\ bibliography. \\ Hemofilia adquirida. Apresentação de caso clínico e revisão da \\ bibliografia.
}

María Fiorella Vuan

ORCID: 0000-0002-9716-2879

Residente Medicina Interna. Hospital

Clínicas. UdelaR.

Marie-Anne Morin

ORCID: 0000-0001-8472-3254

Asistente Clínica Médica. Hospital

Clínicas. UdelaR.

Ximena Añón

ORCID: 0000-0001-7670-773X Internista. Profesora Adjunta Clínica Médica. UdelaR

Victoria Casada

ORCID: 0000-0001-6284-7678

Residente Medicina Interna. Hospital

Clínicas. UdelaR.

Leonardo Sosa

ORCID: 0000-0002-1489-6894 Internista. Profesor Director Clínica Médica. UdelaR.
Resumen: La hemofilia adquirida es un trastorno de la coagulación poco frecuente causado por autoanticuerpos circulantes que inhiben factores de la coagulación, principalmente el F VIII. Un porcentaje considerable de pacientes con hemofilia adquirida mueren a causa de un diagnóstico tardío con el consecuente retraso en el inicio del tratamiento. Los objetivos principales del tratamiento son controlar el sangrado, erradicar el inhibidor y tratar los trastornos subyacentes que se logren identificar. Presentamos el caso de una mujer de 72 años con el antecedente de una artritis reumatoidea que se presentó con un síndrome hemorragíparo de tipo coagulopático de aparición espontánea.

Palabras clave: autoanticuerpos; factor VIII; hemofilia adquirida.

Abstract: Acquired hemophilia is a rare coagulation disorder caused by circulating autoantibodies that inhibit coagulation factors, primarily F VIII. A considerable percentage of patients with acquired hemophilia die due to a late diagnosis with the consequent delay in the start of treatment. The main goals of treatment are to control bleeding, eradicate the inhibitor and treat underlying disorders that can be identified. We present the case of a 72-year-old woman with a history of rheumatoid arthritis who presented with a coagulopathic hemorrhagic syndrome of spontaneous onset.

Key words: autoantibodies; factor VIII; Acquired hemophilia

Resumo: A hemofilia adquirida é um distúrbio raro da coagulação causado por autoanticorpos circulantes que inibem fatores de coagulação, principalmente F VIII. Uma porcentagem considerável de pacientes com hemofilia adquirida morre devido a um diagnóstico tardio com o consequente atraso no início do tratamento. Os principais objetivos do tratamento são controlar o sangramento, erradicar o inibidor e tratar os distúrbios subjacentes que podem ser identificados. Apresentamos o caso de uma mulher de 72 anos com história de artrite reumatoide que apresentou síndrome hemorrágica coagulopática de início espontâneo.

Palavras-chave: autoanticorpos; fator VIII; Hemofilia adquirida

Recibido: 12/01/2020 Aceptado: 28/02/2020

Clínica Médica B. Hospital de Clínicas “Dr. Manuel Quintela”. Facultad de Medicina. Universidad de la República (UdelaR). Montevideo, Uruguay.

Correspondencia: E-mail: mariavuan@gmail.com 


\section{Introducción}

La hemofilia adquirida es un trastorno de la coagulación poco frecuente, con una incidencia estimada de aproximadamente 1.5 casos por millón de personas/año. Es causada por autoanticuerpos circulantes que inhiben factores de la coagulación, principalmente el F VIII ${ }^{(1)}$. (2).

La media de edad al diagnóstico es de 65 años, sin un claro predominio entre ambos sexos

Es considerada idiopática en el $60 \%$ de los casos, estando asociada a enfermedades autoinmunes en un $20 \%$. Dentro de este porcentaje se destaca una asociación con la artritis reumatoidea (AR) en un $4-8 \%$ de los casos ${ }^{(3)}$. Puede también asociarse a neoplasias tanto sólidas como hematológicas, embarazo y transfusiones múltiples. (4)

Determina una alta morbilidad y mortalidad dependiente de varios aspectos: magnitud del sangrado, edad avanzada del paciente, enfermedades subyacentes y efectos secundarios del tratamiento inmunosupresor. ${ }^{(5)}$

Un porcentaje considerable de pacientes con hemofilia adquirida mueren a causa de un diagnóstico tardío con el consecuente retraso en el inicio del tratamiento. En ello radica la importancia de su reconocimiento temprano. ${ }^{(6)}$

A continuación reportamos el caso de una paciente con diagnóstico de AR que presenta un síndrome coagulopático en el que se hace diagnóstico de hemofilia adquirida por la presencia de inhibidor del factor VIII.

\section{Caso clínico}

Sexo femenino, 72 años con antecedentes personales de hipertensión arterial de larga data. AR diagnosticada en 2014, recibió prednisona 20 mg/día desde entonces hasta marzo de 2019. Nunca había recibido tratamiento con fármacos modificadores de la enfermedad.

Consulta por cuadro de 3 meses de evolución dado por equimosis y hematomas de aparición espontánea en miembros superiores, inferiores y tronco. Presentaba al momento de la consulta un extenso hematoma, de aparición espontánea a nivel de miembro superior izquierdo, con dolor en codo e impotencia funcional. No petequias. No sangrados mucosos ni viscerales. Negaba la ingesta de fármacos o tóxicos. Destacamos la suspensión de corticoides al inicio del cuadro. No presentaba elementos secuelares ni de actividad de su AR.

Examen físico evidenciaba un extenso hematoma a nivel de miembro superior izquierdo (figura 1), equimosis y hematomas en tronco y miembros inferiores en diferentes estadios evolutivos. La ecografía de miembro superior derecho confirma la extensión del hematoma comprometiendo el sector muscular externo del bíceps braquial con líquido anecogénico en receso articular posterior.

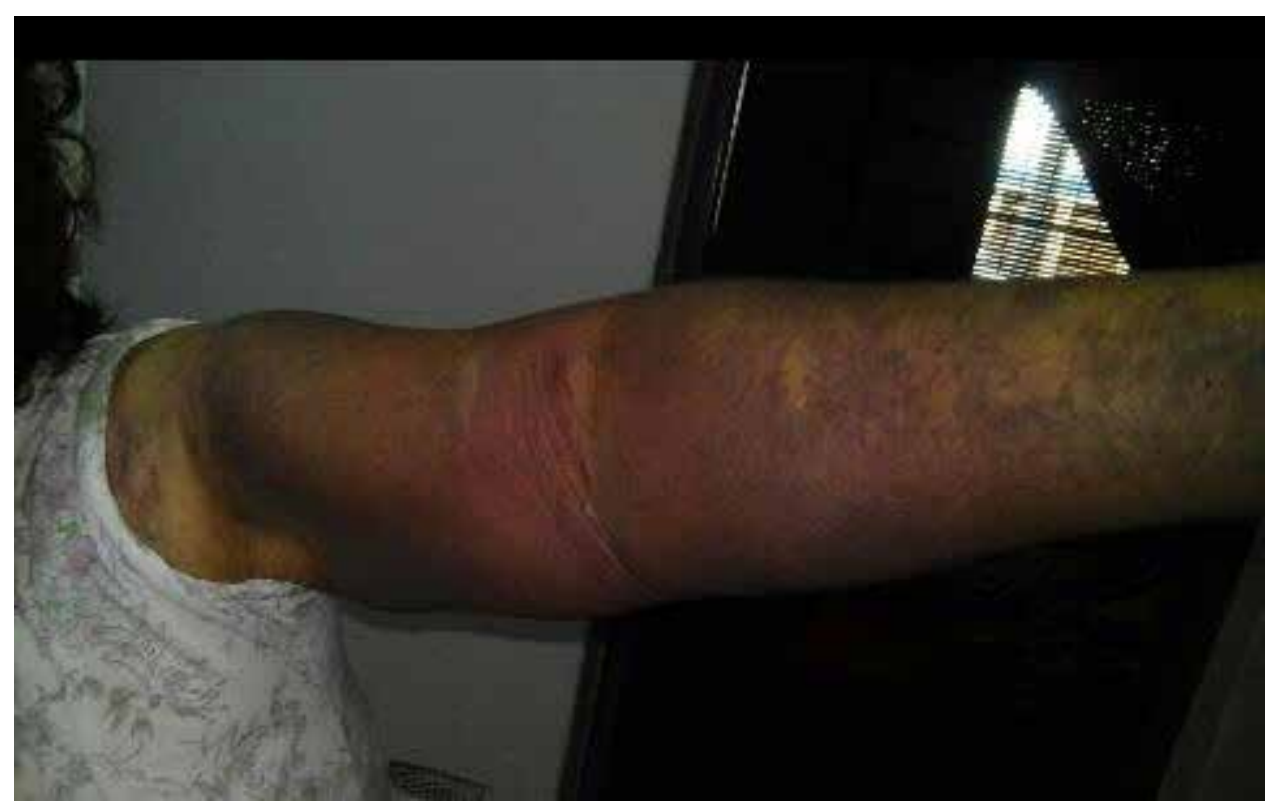


Con planteo de una coagulopatía, se realizan estudios para su confirmación: plaquetas $347.000 / \mathrm{mm} 3$, tiempo de protrombina $80.7 \%$, tiempo parcial de tromboplastina activado (aPTT) - incoagulable. Se realizó la prueba de mezcla con plasma normal, determinando el aPTt sin corrección del mismo. Fibrinógeno 398 mg/DI.

Se procedió a dosificar el Factor VIII encontrándose disminuida su actividad (menor 0.25\%), y se comprobó la presencia de un inhibidor del Factor VIII a altos títulos (1064 Unidades Bethesda).

Con estos hallazgos se realizó diagnóstico de hemofilia adquirida.

Para la valoración etiológica se realizaron estudios para descartar causas infecciosas, inmunes y tumorales. Serología virus hepatitis $B$, virus hepatitis $C, V I H$ y VDRL que fueron no reactivos. Factor reumatoideo $106 \mathrm{UI} / \mathrm{mL}$, anticuerpos antipéptido citrulinado cícclico (anti-CCP) $96 \mathrm{UI} / \mathrm{mL}$, anticuerpos antinucleares (ANA) 1/80, velocidad eritrosedimentación $40 \mathrm{~mm} / 1$ hora. Tomografía computada (TC) de tórax, abdomen y pelvis destaca la presencia nódulo sólido de $8 \mathrm{~mm}$ en lóbulo superior pulmonar izquierdo.

Luego de descartadas las causas infecciosas y neoplásicas se planteó como etiología más probable la autoinmune en el contexto de su AR. En cuanto al nódulo pulmonar evidenciado en la TC, por su tamaño, sus características y tratarse de una paciente no fumadora se está realizando seguimiento del mismo.

Se realizó tratamiento con pulsos de $500 \mathrm{mg}$ de metilprednisolona intravenosa durante 3 días. Continuando con prednisona $60 \mathrm{mg}$ día $(1 \mathrm{mg} / \mathrm{kg} /$ día) en plan de descenso gradual. De la evolución inmediata, se destaca que el título de descenso del inhibidor del Factor VIII era menor al esperado, por lo se inició ciclofosfamida vía oral 100 mg día. En la evolución agregó hematoma a nivel de cara posterior de muslo izquierdo y hemartrosis de rodilla izquierda por lo que recibió concentrado de complejo de protrombina activado (FEIBA) con buena evolución posterior.

Se mantuvo tratamiento ambulatorio con prednisona y ciclofosfamida continuando su control evolutivo con Medicina Interna y Hematología.

Reingresa por un aumento del hematoma a nivel de cara posterior de muslo izquierdo, recibe nuevamente tratamiento con FEIBA manteniéndose estable, sin aumento del mismo.

\section{Discusión}

La hemofilia adquirida es un trastorno hemorrágico de mecanismo inmune causado por la aparición de autoanticuerpos específicos que inhiben factores de la coagulación. El FVIII es la diana más frecuente de estos autoanticuerpos. ${ }^{(7)}$

Sin embargo, se han descrito anticuerpos contra otros factores de la coagulación con limitados reportes en la literatura.

La edad media de aparición es de 65 años, aunque presenta una distribución bifásica. Un primer pico representado por mujeres jóvenes (principalmente en el puerperio o en contexto de enfermedades autoinmunes). El otro pico afecta a pacientes mayores de 60 años sin claras diferencias de sexo. ${ }^{(8)}$

Es una enfermedad potencialmente mortal por el riesgo de sangrados fatales. Los sitios de sangrado más comunes incluyen músculos, piel, mucosas y tejidos blandos. ${ }^{(9)}$

Está vinculada a diferentes condiciones. Se ha observado que la asociación con enfermedades autoinmunes representa hasta un $18 \%$ de los casos. Las asociaciones específicas incluyen lupus eritematosos sistémico, AR, esclerosis múltiple y afecciones dermatológicas como la psoriasis y el pénfigo vulgar, entre otras. Se ha visto que estos pacientes tienden a desarrollar un mayor título de inhibidor del factor VIII, y es frecuente que requieran de tratamientos más agresivos para la erradicación del mismo. ${ }^{(10)}$

La asociación con el embarazo está bien establecida y representa aproximadamente el 10\% de todos los casos. Ocurre sobre todo en mujeres primíparas dentro de los primeros 3 meses posteriores al parto y pueden provocar una hemorragia uterina grave ${ }^{(10,11)}$.

Otra comorbilidad frecuentemente asociada son las neoplasias malignas, incluso en estadios preclínicos. Se incluyen tanto tumores sólidos como neoplasias hematológicas. ${ }^{(12)}$ 
Dentro de las enfermedades hematoncológicas más comúnmente asociadas se identifican sobre todo aquellas relacionadas a una alteración inmunitaria, tales como la leucemia linfoide crónica, el linfoma no Hodgkin, el mieloma múltiple y la macroglobulinemia de Waldenström. Las neoplasias sólidas más frecuentemente asociadas son el cáncer de próstata y de pulmón. ${ }^{(11)}$

La sospecha diagnóstica se confirma con las siguientes pruebas de laboratorio:

1) Tiempo de tromboplastina parcial activado (aPTT) prolongado, que no se corrige con plasma normal.

2) Disminución de las concentraciones de factor VIII.

Detección de anticuerpos anti factor VIII. ${ }^{(13)}$

Lo más frecuente es el hallazgo aislado de un aPTT prolongado, con tiempo de protrombina, tiempo de trombina y recuento plaquetario normales. ${ }^{(13)}$

Esta prolongación del aPTT puede deberse tanto a una deficiencia de uno de los factores de la coagulación de la vía intrínseca (FVIII, IX, XI o XII) como a la presencia de un inhibidor de los mismos. Para distinguir la deficiencia de un factor de la existencia de un inhibidor, se debe realizar la prueba de mezclas con plasma normal. El aPTT corregirá a su valor normal si existe deficiencia, de lo contrario el FVIII del plasma normal será inhibido y el aPTT no se corregirá. (3)

Si el aPTT no corrige, a continuación se debe determinar el nivel de actividad del factor VIII. Si la actividad se encuentra disminuida, se debe proseguir a identificar el anticuerpo anti factor VIII (inhibidor del factor VIII). (3)

Existen varios test para la detección y cuantificación de autoanticuerpos inhibidores; el más utilizado es el método Bethesda. ${ }^{(7)}$

El test se basa en la estimación de la neutralización de una concentración conocida de FVIII que un inhibidor del mismo produce durante un período de tiempo determinado a $37 \rrbracket$ C. El inhibidor se cuantifica en unidades Bethesda (UB), sabiendo que una Unidad Bethesda corresponde a la cantidad de inhibidor capaz de bloquear al $50 \%$ del FVIII circulante. ${ }^{(14)}$

El número y gravedad de las hemorragias no están relacionados ni con los niveles de FVIII residual ni con el título del inhibidor.

Los objetivos principales del tratamiento de la hemofilia adquirida son controlar el sangrado, erradicar el inhibidor, tratar los trastornos subyacentes que se logren identificar y evitar que el paciente sufra traumatismos o sea expuesto a procedimientos invasivos. ${ }^{(15)}$

Las opciones para el control hemostático son el uso de agentes con efecto bypass y estrategias que ayuden a elevar la actividad del F VIII. ${ }^{(16)}$

Los agentes con efecto bypass son los más comúnmente usados como primera línea de tratamiento, tanto el factor Vlla recombinante ( $\mathrm{rFVlla)}$ como los concentrados de complejo protrombínico activado (CCPa). ${ }^{(17)}$

El rFVIla favorece la generación de fibrina mediante mecanismos en los que predomina la vía extrínseca de la coagulación, la cual, al no requerir la participación del FVIII evita que el inhibidor dificulte la generación de trombina. EL CCPA aporta factores activados capaces de obviar la presencia del FVIII para producir trombina. ${ }^{(7)}$

La elección entre ambas estrategias dependerá del sitio y gravedad del sangrado, así como las características del paciente y la posibilidad de acceso al fármaco. ${ }^{(17)}$

Si bien los agentes hemostáticos se administran para controlar la hemorragia, también es necesario el inicio de tratamiento inmunosupresor con el objetivo de suprimir la producción del anticuerpo inhibidor. Se recomienda el inicio terapia inmunosupresora tan pronto como se establezca el diagnóstico de hemofilia adquirida. ${ }^{(18)}$

El tratamiento habitual incluye la administración de glucocorticoides en monoterapia, de elección prednisona a dosis de $1 \mathrm{mg} / \mathrm{kg}$ o asociado a ciclofosfamida oral en dosis de 50-100 $\mathrm{mg} /$ día. $^{\left({ }^{(1)}\right.}$ Se recomienda la asociación si el paciente ya ha sido tratado con glucocorticoides por alguna otra condición. ${ }^{(20)}$

De no existir respuesta al tratamiento instituido luego de 4 a 6 semanas, el tratamiento con rituximab se considera una alternativa de segunda línea. ${ }^{(20)}$ 
Se debe tener en cuenta que la mayoría de los pacientes corresponden a una población de edad avanzada con comorbilidades, por lo que los regímenes terapéuticos deberán apuntar a equilibrar la necesidad de una rápida erradicación del inhibidor y la exposición a efectos adversos de la terapia inmunosupresora. ${ }^{(20)}$

\section{Conclusiones}

La rápida sospecha y el diagnóstico temprano de la hemofilia adquirida son de vital importancia en vistas a realizar un tratamiento oportuno con el objetivo de evitar sangrados graves.

Este diagnóstico conllevará al estudio de condiciones asociadas (neoplasias o enfermedades autoinmunes) y podrá revelar una enfermedad subyacente, cuyo tratamiento será parte de los objetivos para el control de la coagulopatía.

La asistencia deberá llevarse a cabo por un equipo multidisciplinario integrado por internista, hematólogo y especialistas en Hemoterapia y Medicina Transfusional, en vistas al seguimiento evolutivo y manejo de complicaciones.

\section{Agradecimientos}

A la Cátedra de Hemoterapia y Medicina Transfusional.

\section{Bibliografía}

1- Franchini M, Vaglio S, Marano G, Mengoli C, Gentili S, Pupella S, et al. Acquired hemophilia A: a review of recent data and new therapeutic options. Hematology. 2017; 22(9): 514-520.

2- Ghozlani I, Mounach A, Ghazi M, Kherrab A, Niamane R. Targeting Acquired Hemophilia A with Rheumatoid Arthritis by a Rituximab Shot: A Case Report and Review of the Literature. Am J Case Rep. 2018 May; 19: 582-588.

3- Mulliez S, Vantilborgh A, Devreese K. Acquired hemophilia: A case report and review of the literature. Int J Lab Hematol. Jun 2014; 36(3): 398-407.

4- Kessler CM, Knobl P. Acquired haemophilia: An overview for clinical practice. Eur J Haematol. Dec 2015; 95(81): 36-44.

5- Collins W, Percy L. Advances in the understanding of acquired haemophilia A: implications for clinical practice. British Journal of Haematology. 2009 Dec; 148, 183-194.

6- Drobieckia A, Pasiarskia M, Husb I, Sokołowskab B, Wateka M. Acquired hemophilia in the patient suffering from rheumatoid arthritis: case report. Blood Coagulation \& Fibrinolysis. 2013 Dec; 24(8): 874-880.

7- Mingot-Castellano M, Núnez R, Rodríguez-Martorell F. Hemofilia adquirida: epidemiología, clínica, diagnóstico y tratamiento. Medicina Clínica 2017; 148(7): 314-322.

8- Borg JY, Guillet B, Le Cam-Duchez V, Goudemand J, Lévesque H. Outcome of acquired haemophilia in France: The prospective SACHA (Surveillance des Auto antiCorps au cours de l'Hémophilie Acquise) registry. Haemophilia. 2013 Jul; 19(4): 564-70.

9- Kimura K, Kuriyama A, Kuninaga N, Sasaki A. Acquired Hemophilia. Intern Med. 2015 April. 54(7): 865, 2015.

10- Sborov DW, Rodgers GM. Acquired hemophilia A: A current review of autoantibody disease. Clin Adv Hematol Oncol. 2012 Jan; 10(1): 19-27.

11- Franchini M, Gandini G, Di Paolantionio T, Mariani G. Acquired hemophilia A: a concise review. Am J Hematol. 2005 Sep; 80(1): 55-63.

12- Shetty S, Bhave M, Ghosh K. Acquired hemophilia A: diagnosis, aetiology, clinical spectrum and treatment options. Autoimmun Rev. 2011 Apr; 10(6): 311-316.

13- Benardete-Harari D, Sánchez-Cárdenas A, Meraz-Ávila D, Nellen-Hummel H, Halabe-Cherem J. Hemofilia adquirida. Una causa rara de hemorragia obstétrica. Med Int Méx 2015 Jan; 31: 174-180.

14- Sahud MA. Factor VIII inhibitors. Laboratory diagnosis of inhibitors. Semin Thromb Hemost. 2000; 26(2): 195-203. 
15- Franchini M, Gandini G, Di Paolantionio T, Mariani G. Acquired hemophilia A: a concise review. Am J Hematol. 2005 Sep; 80(1): 55-63.

16- Hay CRM, Brown SA, Collins PW, Keeling DM, Liesner R. The diagnosis and management of factor VIII and IX inhibitors: a guideline from the United Kingdom Haemophilia Centre Doctors Organisation. Br J Haematol 2006 May; 133: 591-605.

17- Collins PW. Treatment of acquired hemophilia A. J Thromb Haemost 2007; 5: 893-900.

18- Collins PW, Hirsch S, Baglin TP, Dolan G, Hanley J, Makris M, et al. Acquired haemophilia A in the UK: a two year national surveillance study by UK Haemophilia Centre Doctors Organisation. Blood 2007 Mar; 109 (5): 1870-7.

19- Giangrande P. Hemofilia adquirida. Tratamiento de la hemofilia. 2012 Nov. 38: 3-4.

20- Huth-Kühne A, Baudo F, Collins P, Ingerslev J, Kessler C et al. International recommendations on the diagnosis and treatment of patients with acquir ed haemophilia. Haematologica 2009; 94(4): 566-575.

\section{Aporte cada autor al trabajo}

María Fiorella Vuan: concepción y diseño del trabajo, redacción del manuscrito.

Marie-Anne Morin: concepción y diseño del trabajo, revisión crítica del manuscrito.

Ximena Añón: revisión crítica del manuscrito.

Victoria Casada: concepción y diseño del trabajo.

Leonardo Sosa: concepción y diseño del trabajo, revisión crítica del manuscrito. 